athletes. Computed tomography Coronary Angiography (CTCA) is an imaging modality of choice for identification and characterization of coronary artery anomalies. The prevalence of AAOCA is usually cited as $1 \%-2 \%$ of the general population and also reported at $0.8 \%-1.3 \%$ in invasive angiographic studies and $1 \%-5 \%$ in reported CTCA series. CTCA service is established in West of Scotland since 2015 and no data has been published about prevalence of AAOCA nor any directly related adverse cardiac events formally studied in these patients here.

Methods Patients with anomalous aortic origin of coronary artery (AAOCA) were identified in the CTCA series between February 2015 and October 2020 in a large tertiary referral centre that caters to most CTCA referrals in West of Scotland. The electronic medical records of these patients were retrospectively checked between February 2015 and February 2021 with a standard evaluation questionnaire and data was independently reviewed by the authors.

Results A total of 2840 patients' electronic records were evaluated and 79 patients with AAOCA were identified. There were 57 males (72\%) and 22 Females (28\%). The mean age was 47.4 years for males and 51.3 years for females. Out of 79 AAOCA, 59\% were anomalous right coronary artery, $29 \%$ were anomalous left circumflex, 7\% were anomalous left main coronary artery and 2\% were anomalous Left anterior descending coronary artery. $86 \%$ were referred for symptom of chest pain (deemed atypical in 94\% of these patients with further evidence of negative or inconclusive ETT), 6\% had arrhythmia (no sudden cardiac death or ventricular arrhythmias were recorded), unexplained dyspnoea in $4 \%$, Transient Loss of consciousness (no CPR needed for recovery) in $4 \%$ of patients. High risk (intra-mural, intramyocardial, high origin or inter-arterial course) of AAOCA was noted in $47 \%$ patients $(n=37 ; 84 \%$ originated from a different coronary sinus and $16 \%$ directly from another coronary artery). Obstructive coronary disease (CAD-RADS score $=3$ and above) was noted in only 9\% of AAOCA $(n=7)$ compared to $29 \% \quad(n=23)$ in non-anomalous coronary arteries. All AAOCA with obstructive disease were noted to be further referred for functional assessment of ischaemia or invasive coronary angiography. Only $14 \% \quad(n=5$ out of 37$)$ with a high risk AAOCA course were referred by the clinicians for functional assessment of ischaemia or arrhythmia and the results were benign. There were 4 deaths noted in this observed cohort and none of them were directly related to coronary artery disease.

Conclusions The observed prevalence of AAOCA in this CTCA series is $2.8 \%$ in West of Scotland and noted to be higher in males $(\mathrm{p}<0.0001)$. The prevalence of obstructive coronary artery disease in AAOCA was relatively low compared to non-AAOCA group $(\mathrm{p}<0.001)$. High risk (intramural, intra-myocardial, high origin or inter-arterial) course of AAOCA was significant among those identified with AAOCA $(p<0.001)$ and $1.3 \%$ of whole study cohort. The rate of further functional assessment of such high risk AAOCA was noted to be low. No sudden cardiac deaths were noted and no directly AAOCA related mortality was noted. The data is limited by findings of AAOCA in patients that were mostly referred for 'rule out' CTCA investigation for low risk clinical cardiac symptoms. Further functional assessment and follow up of patients identified with high risk course of AAOCA is recommended as per current guidelines.

Conflict of Interest None
169 REAL WORLD NHS EXPERIENCE OF CTCA WITH FFRCT FOR THE DETECTION OF SURGICAL CORONARY ARTERY DISEASE - THE CASE FOR ENHANCED PRE-PROCEDURAL PLANNING?

${ }^{1}$ David Murphy, ${ }^{1}$ John Graby, ${ }^{2}$ Tushar Rakhecha, ${ }^{2}$ Jessica Donaghue, ${ }^{2}$ Dan McKenzie, ${ }^{2}$ Sri Raveen Kandan, ${ }^{2}$ Ali Khavandhi, ${ }^{2}$ Kevin Carson, ${ }^{1}$ Rob Lowe, ${ }^{2}$ Ben Hudson, ${ }^{1} J o n a t h a n$ Rodrigues. ${ }^{1}$ Royal United Hospitals Bath NHS Foundation Trust, Bath, UK; ${ }^{2} R U H$ Bath

\subsection{6/heartjnl-2021-BCS.166}

Introduction CT coronary angiography (CTCA) with fractional flow reserve (FFRCT) is a key diagnostic tool in the guidance led evaluation of chronic coronary syndrome (CCS). A small percentage of those patients presenting with CCS will have a pattern of coronary artery disease (CAD) where they would be better served with surgical revascularisation. CTCA alone is increasingly used to rule out important CAD pre-valvular surgery in the absence of an invasive coronary angiogram (ICA). Thus, this study tested to see if CTCA with FFRCT was sufficient to predict surgical CAD relative to subsequent ICA findings.

Methods This retrospective single-centre study analysed all patients with CCS who underwent a CTCA with FFRCT, where findings led to a subsequent ICA from August 2018 to January 2021. Those patients who had significant left main stem (LMS) and/or flow limiting disease in three major epicardial blood vessels were included (3VD). Flow limiting disease was defined as an FFRCT of $\leq 0.8$ ( $2 \mathrm{~cm}$ distal to the stenosis) in the left anterior descending (LAD), circumflex (LCx), principle obtuse marginal (OM) or right coronary artery (RCA). This was then compared to the ICA where significance was defined as a stenosis $>50 \%$ for the LMS and $>70 \%$ for the other epicardial vessels and/or iFR of $\leq 0.89$ or FFR $\leq 0.8$.

Results A total of 565 patients had a CTCA with FFRCT, of which 164 had a subsequent ICA with sufficient data for analysis and 35 of these patients met inclusion criteria (LMS disease only $7 / 35$, 3VD 25/35 and both LMS and 3VD 3/35 on CTCA with FFRCT). Relative to ICA the overall sensitivity, specificity, positive predictive value, negative predictive value and accuracy of CTCA and FFRCT for predicting surgical CAD was 83\% (95\% CI 61-95), 92\% (95\% CI 86-96), 61\% (95\% CI 47-74), 97\% (95\% CI 93-99) and 90\% (95\% CI $85-94)$ respectively.

Conclusion CTCA with FFRCT was insufficient for a direct decision on surgical revascularisation in this cohort, particularly given the different risk profiles of ICA, PCI and bypass surgery. Importantly, however, the performance of CTCA with FFRCT for detection of surgical CAD would enable enhanced pre-procedural planning. This includes providing an opportunity to counsel patients in more detail on potential findings and their preference if a surgical pattern of disease is confirmed, consider pre-ICA MDT discussion, and ensure likely complex, high-risk cases are placed on an appropriate list.

Conflict of Interest None

\section{SPECT MYOCARDIAL CORONARY FLOW RESERVE - THE INITIAL GLASGOW EXPERIENCE}

${ }^{1}$ Nicholas Goodfield, ${ }^{1}$ Richard McFarlane, ${ }^{2}$ Craig Paterson, ${ }^{2}$ Jamie Robinson, ${ }^{2}$ Kirsty Jones, ${ }^{2}$ Kurian Oommen, ${ }^{2}$ Robin McDade. 'Dept of Cardiology, Glasgow Royal Infirmary, Glasgow, UK; ${ }^{2}$ Dept of Nuclear Cardiology, Glasgow Royal Infirmary

10.1136/heartjnl-2021-BCS.167 
Stress Protocol
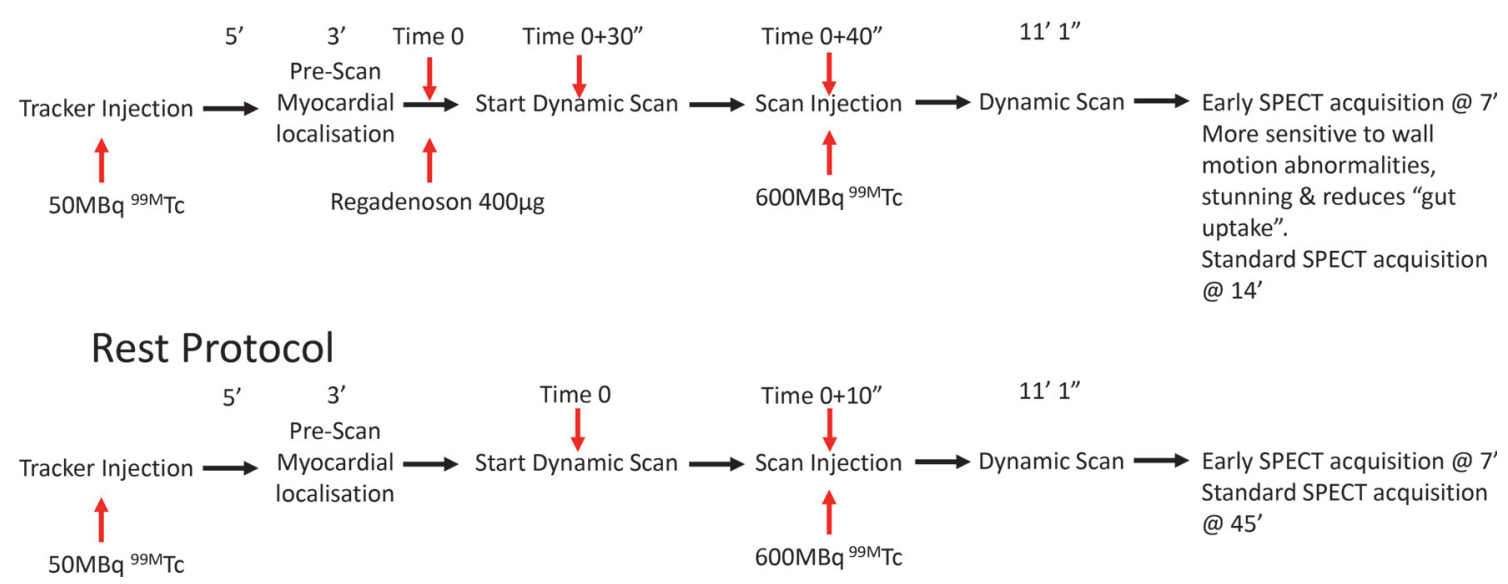

\section{Abstract 170 Figure 1}

Introduction Identification of myocardial ischaemia \& the need for mechanical intervention has increasingly become dependent on measurements of coronary artery flow in terms of FFR, CMR \& IMR at invasive coronary angiography \& coronary flow reserve (CFR) at CTCA, in addition to anatomy \& standard perfusion scanning. CFR measured by PET imaging has been available for some time $\&$ has shown additional prognostic value over myocardial perfusion imaging alone. Unfortunately, PET has limited clinical availability. The advent of solid state gamma cameras has increased count sensitivity \& temporal resolution. Thus, it is possible to perform measurements of global \& regional CFR \& myocardial blood flow (MBF) from a dynamic SPECT acquisition. Adding CFR measurements to a SPECT myocardial perfusion scan may be particularly useful for patients with triple vessel disease or suspected microvascular angina $((\mu \mathrm{VA})$ with so called 'matched' defects. The measurement requires dynamic imaging with stress \& at rest, from the moment of injection of myocardial perfusion tracer - Diagram 1 Detailed tissue kinetic modelling is used to estimate absolute MBF \& CFR. The dynamic data is processed using a Leppo net retention model on the GE Alcyone software suite. The Renkin-Crone flow model is used to convert the retention ratio to MBF. CFR is then calculated as the ratio of hyperaemic to rest absolute $\mathrm{MBF}$.

Results Table 1We have now performed 49 SPECT MPS's with flow measurements, as part of clinical service, on a GE Discovery NM530c scanner.Reason for referral was divided into possible $\mu \mathrm{VA}, \mathrm{ACP} \& \mathrm{TCP}$. Of those with possible $\mu \mathrm{VA}$, not all had had coronary angiography to support the diagnosis. However, whilst most had normal standard myocardial perfusion scans, half had evidence of abnormal CFR supporting the potential diagnosis in these cases $\&$ increasing the identification of potential ischaemia from 20\% with standard MPS to $80 \%$ with CFR.In patients with ACP, the addition of CFR to standard SPECT MPS, had a lesser impact, increasing the suggestion of ischaemia from $23 \%$ to $35 \%$.In patients with TCP, although $60 \%$ of previous angiograms had been normal, again the addition of CFR increased identification of potential ischaemia from $23 \%$ of patients using standard SPECT MPS, to $72 \%$ with CFR assessment.

Conclusions The addition of non-invasive flow assessment to standard SPECT MPS increases the identification of potential ischaemia in patients with potential $\mu \mathrm{VA}$ or TCP, presumably due to identifying flow reduction in 'matched' ischaemic

\begin{tabular}{lclll}
\multicolumn{4}{l}{ Abstract 170} & Table 1 \\
\hline Referral Reason & $\mathrm{n}$ & iCA / CTCA & Standard SPECT MPS & Flow Assessment \\
$\mu$ VA & 10 & Normal 8 & Normal 8 & Normal 5 \\
& & & Abnormal 2 & Abnormal 5 \\
Atypical CP (ACP) & 17 & Normal 6 & Normal 13 & Normal 11 \\
& & Abnormal 5 & Abnormal 4 & Abnormal 6 \\
Typical CP (TCP) & 22 & Normal 12 & Normal 17 & Normal 6 \\
& & Abnormal 8 & Abnormal 5 & Abnormal 16 \\
\hline
\end{tabular}

defects which may be missed on standard perfusion scanning. However, whilst CFR has less advantages in terms of additional information in patients with ACP, it may increase confidence in the exclusion of ischaemia.

Conflict of Interest none

\section{THE ROLE OF ECHOCARDIOGRAPHY IN IMPLANTABLE CARDIAC ELECTRONIC DEVICE INFECTION}

${ }^{1}$ Alice Cowley, ${ }^{2}$ Sam Straw, ${ }^{2} J a m e s$ O'Neill, ${ }^{2}$ Anshuman Sengupta, ${ }^{2}$ Wazir Baig, ${ }^{2}$ Jonathon Sandoe. ${ }^{1}$ Nottingham University Hospitals, Nottingham, UK; ${ }^{2}$ Leeds Teaching Hospital Trust

\subsection{6/heartjnl-2021-BCS.168}

Introduction The use of implantable cardiac electronic devices (ICEDs) is rising in part due to expanding indications and an aging population [1]. ICED infection occurs in $0.5-2.2 \%$ of all implants [1] and implicated in around 10\% of cases of infective endocarditis (IE) [2]. ICED infection is associated with prolonged antibiotic therapy and hospital stays, with a mortality rate as high as $35 \%[1,3,4]$. Echocardiography has an established role in the diagnosis of ICED infection and is advocated by guidelines [1], however whether imaging can guide duration of antimicrobial therapy is unclear.

Methods We aimed to determine whether echocardiography following ICED extraction was associated with length of antibiotic therapy. Consecutive patients undergoing ICED extraction at Leeds Teaching Hospitals between 01/01/2006 and 31/ $12 / 2017$ were identified from a prospectively maintained IE database. Patient demographics, microbiology and echocardiographic data were recorded. 